\title{
An Adaptive Finite Element Method for Linear Elliptic Problems
}

\author{
By Kenneth Eriksson and Claes Johnson
}

\begin{abstract}
We propose an adaptive finite element method for linear elliptic problems based on an optimal maximum norm error estimate. The algorithm produces a sequence of successively refined meshes with a final mesh on which a given error tolerance is satisfied. In each step the refinement to be made is determined by locally estimating the size of certain derivatives of the exact solution through computed finite element solutions. We analyze and justify the algorithm in a model case.
\end{abstract}

Introduction. Recently, adaptive finite element methods for elliptic problems have attracted much interest, see, e.g., [1]-[4], [6], [7], [13], and are rapidly becoming increasingly important in applications. The basic problem concerning such adaptive methods is roughly the following: Given an elliptic problem with no a priori knowledge of the behavior of the exact solution and a finite element method for this problem together with an error tolerance $\delta>0$ and a certain norm, construct an automatic procedure for finding a finite element mesh such that the error in the corresponding finite element solution is at most $\delta$ in the given norm. One further requires the constructed mesh to be efficient in the sense that, e.g., the number of elements is nearly minimal. A typical adaptive procedure could be expected to involve a sequence of finite element solutions on successively refined meshes (starting with, e.g., a quasi-uniform mesh), and the procedure would end when the error is smaller than or equal to the given tolerance. At each step of the procedure an estimate of the error on the given mesh would be made, and in case the error tolerance is not met, a refined mesh to be used in the next step would be constructed. Typically, the procedure would generate meshes which are refined in regions where the exact solution is nonsmooth such as, e.g., neighborhoods of corners in a polygonal domain. In the methods proposed by Babuskka and coworkers [2]-[4], the error estimate at each step is based on solving local problems involving a local residual, and the refinements are carried out according to the size of the solutions of the local problems. This method seems to produce reasonable meshes in many cases but appears to be difficult to theoretically justify in several dimensions (cf. [2], [4]).

The purpose of this note is to present and analyze, in a model case, an adaptive procedure which is based on a different approach than the Babuska method. As a model problem we shall consider the Poisson equation

$$
\left\{\begin{aligned}
-\Delta u=f & \text { in } \Omega, \\
u=0 & \text { on } \Gamma,
\end{aligned}\right.
$$

Received June 24, 1985; revised October 16, 1986.

1980 Mathematics Subject Classification (1985 Revision). Primary 65N15, 65N30. 
in a bounded domain $\Omega$ in the plane with boundary $\Gamma$. We shall consider the standard finite element method for $(0.1)$ using continuous piecewise linear functions on a triangulation $T_{h}=\{K\}$ of $\Omega$ into triangles $K$ of diameter $h_{K}$. We shall assume that we want to control the gradient of the error in the maximum norm (cf. Remark 1.2 below). The error control will be based on an optimal a priori estimate of the form (cf. [17], [8])

$$
\left\|\nabla\left(u-u^{h}\right)\right\|_{\infty, \Omega} \leq C_{0} \max _{K \in T_{h}} h_{K}|u|_{2, \infty, K},
$$

where for $m=0,1, \ldots$, and $\omega$ a domain,

$$
|v|_{m, \infty, \omega}=\sup _{\substack{x \in \omega \\|\alpha|=m}}\left|D^{\alpha} v(x)\right|, \quad\|v\|_{\infty, \omega}=|v|_{0, \infty, \omega}
$$

and where $u^{h}$ denotes the finite element solution. Here we use the usual multi-index notation $D^{\alpha} v$ for derivates of order $|\alpha|$. Further, $C_{0}$ denotes a positive constant assumed for the moment to be known approximately (the problem of roughly estimating $C_{0}$ is commented on in Remark 3.1 below). Given now a tolerance $\delta>0$, we want to find a finite element solution $u^{h}$ satisfying $\left\|\nabla\left(u-u^{h}\right)\right\|_{\infty, \Omega} \leq \delta$, and thus (0.2) leads us to the following choice of the local mesh size $h_{K}$ :

$$
C_{0} h_{K}|u|_{2, \infty, K} \sim \delta
$$

The obvious idea is now to seek to estimate the quantity $|u|_{2, \infty, K}$ by using computed finite element solutions $u^{h}$ and then determine the local mesh size according to (0.3). We shall present below an algorithm for error control and adaptive mesh selection based on this approach. We shall then consider a model situation where the exact solution has a singularity in $\Omega$ of a certain form, and we shall in this very special case verify that the proposed algorithm will generate a sequence of meshes leading to a final correctly refined mesh on which the error tolerance is met. The basic technical tool to prove this result is a localized version of the a priori estimate (0.2). Using this estimate we prove that it is possible to locally estimate with sufficient accuracy the desired quantity $|u|_{2, \infty, K}$ by using certain (local) difference quotients of computed gradients of $u^{h}$. Thus, we may say that our adaptive algorithm is based on an optimal a posteriori error estimate of the form (0.2) with $|u|_{2, \infty, K}$ replaced by an approximation obtained through the computed solution $u^{h}$. We are presently developing this approach also for adaptive mesh control in time and space for parabolic problems ([10], [11], [14], [15]) and hyperbolic problems ([12]).

The analysis of this note, in which we consider for simplicity the case of an interior singularity on a smooth domain, can be extended to cover problem $(0.1)$ with $\Omega$ a corvex polygonal domain (and $f$ smooth), in which case the exact solution has singularities of strength $r^{\beta}, \beta>1$, at the corners, see Eriksson [9], where also further extensions to nonconvex polygonal domains corresponding to $\beta>\frac{1}{2}$ are given.

The general idea of basing an adaptive method on estimating the derivatives of the exact solution through computed approximate solutions of course is not new and has been used extensively in an intuitive, qualitative only and nonautomatic way in engineering computations. An early paper proposing to base an adaptive method on an energy norm error estimate and to estimate the derivatives of the 
exact solution involved through computations, is given by [7]. A similar approach was also taken in [6] and [16]. Our method is based on the same idea but we extend the setting by considering different norms (cf. Remark 1.2 below), by seeking to justify the algorithm theoretically and also by considering the problem of estimating e.g. the constant $C_{0}$ in (0.2) to make the error control fully quantitative, cf. Remark 3.1 below.

Extensions of the presented results to more general elliptic problems, for instance variable coefficient linear problems, or to higher-order finite elements, seem to be possible. As soon as we have a sharp error estimate together with a local maximum norm error estimate at hand, there is a possibility of using this as a basis for an adaptive procedure. In particular, this means that error estimates which have been considered to be of mostly theoretical interest, in fact may be of key importance in the practical implementation of the finite element method on real life problems in the future!

An outline of this note is as follows. In Section 1 we present the adaptive algorithm. In Sections 2 and 3 we analyze this algorithm in a model case and prove that in this case it performs as desired. Finally, in Section 4 we present the results of some numerical experiments with a particular implementation of the algorithm which show that indeed the algorithm performs in practice as expected.

We shall assume that all finite element meshes $T_{h}$ that occur satisfy a minimum angle condition, i.e., we assume that there is a positive constant $\theta$ such that all angles of all $K \in T_{h}$ for all $T_{h}$ are greater than or equal to $\theta$. Below, we will by $c$ and $C$ denote various positive constants which will be independent of the meshes $T_{h}$ and thus of the corresponding finite element solutions $u^{h}$. The constants may depend on the minimal angle $\theta$, the domain $\Omega$ and on the exact solution $u$ (more precisely on the constants $c$ and $C$ in (2.1)).

1. The Adaptive Algorithm. We shall consider the following standard finite element method for the Poisson equation (0.1): Given the finite element mesh $T_{h}=\{K\}$, find $u^{h} \in V_{h}$ such that

$$
a\left(u^{h}, v\right)=(f, v) \quad \forall v \in V_{h}
$$

where $V_{h}$ is the space of piecewise linear continuous functions $v$ on the triangulation $T_{h}$ vanishing on $\Gamma$, and

$$
a(v, w)=\int_{\Omega} \nabla v \cdot \nabla w d x, \quad(v, w)=\int_{\Omega} v w d x
$$

We assume that we start with a quasi-uniform mesh $T_{\bar{h}}=\{K\}$ with elements $K$ satisfying $c \delta \leq c \bar{h} \leq h_{K} \leq C \bar{h}$. To take $\bar{h} \geq c \delta$ is reasonable since otherwise the initial mesh would be unnecessarily fine in areas where the solution is smooth.

To compute approximations of the derivatives $D^{\alpha} u$ with $|\alpha|=2$ locally, we shall apply certain difference operators $D_{H}^{\gamma}$ to the computed gradient $\nabla u^{h}$. The difference operators $D_{H}^{\gamma}$ will be of the form

$$
D_{H}^{\gamma} v(x)=\frac{v(x \pm \gamma H)-v(x)}{H}
$$

with $\gamma=(1,0)$ or $\gamma=(0,1)$. Here, $H=C h_{K}$ if $x \in K$, and $C$ is a sufficiently large constant, the choice of which will be made precise below. If $x$ is close to the 
boundary, the point $x \pm \gamma H$ is chosen so as to belong to $\Omega$. We will thus use firstorder difference operators $D_{H}^{\gamma}$ involving translations of size $H$ which will typically be of the order of a couple of local mesh widths.

The algorithm can now be formulated as follows:

$1^{\circ}$. Choose $T_{h}=T_{\bar{h}}$ where $T_{\bar{h}}$ is the initial quasi-uniform mesh.

$2^{\circ}$. Given a mesh $T_{h}$, compute the corresponding finite element solution $u^{h} \in$ $V_{h}$.

$3^{\circ}$. Compute the following quantity for each $x \in K \in T_{h}$ :

$$
D_{H}^{2} u^{h}(x)=\max \left\{\left|D_{H}^{\gamma} D^{\alpha} u^{h}(y)\right|:|\alpha|=|\gamma|=1,|y-x| \leq \bar{C} \underline{h}\right\},
$$

where $\underline{h}=\min _{K \in T_{h}} h_{K}$ and $\bar{C}$ is a sufficiently large constant.

$4^{\circ}$. If for all $K \in T_{h}$ we have

$$
C_{0} h_{K} D_{H}^{2}\left(u^{h} ; K\right) \leq \delta
$$

where

$$
D_{H}^{2}\left(u^{h} ; K\right)=\max _{x \in K} D_{H}^{2} u^{h}(x),
$$

then stop and accept the finite element solution $u^{h}$. If not, construct a new mesh $\tilde{T}_{h}$ by minimally refining the old mesh $T_{h}$ so that for each $K \in T_{h}$

$$
C_{0} h_{\tilde{K}} D_{H}^{2}\left(u^{h} ; K\right) \leq \delta \quad \forall \tilde{K} \in \tilde{T}_{h} \text { with } \tilde{K} \subseteq K .
$$

Then redefine $T_{h}=\tilde{T}_{h}$ and return to $2^{\circ}$.

Remark 1.1. Note that to compute $D_{H}^{2}\left(u^{h} ; K\right)$, only local simple computations are involved; cf. Section 4 below for the particular implementation of the algorithm used in the numerical experiments. The quantity $D_{H}^{2}\left(u^{h} ; K\right)$ is basically to be thought of as an approximation of $|u|_{2, \infty, K}$. For technical reasons, $D_{H}^{2}\left(u^{h} ; K\right)$ involves difference quotients at points in an $\underline{h}$-neighborhood of $K$. Variants of this procedure are possible. For instance, we may take $\bar{C}=0$ in (1.2) and avoid the maximization if we require the meshes $T_{h}$ to have a certain "stiffness", guaranteeing that the mesh size does not change too quickly (see [9]).

Remark 1.2. One may choose to control the error in norms other than the $|\cdot|_{1, \infty, \Omega}$ norm used in (0.2). For example, we may take as starting point a maximum norm estimate of the form (cf., e.g., [8], [17], [18], [19])

$$
\left\|u-u^{h}\right\|_{\infty, \Omega} \leq C_{0} \max _{K \in T_{h}} h_{K}^{2}|u|_{2, \infty, K},
$$

where the constant $C_{0}$ here also includes a logarithmic dependence of $\min h_{K}$, or the standard energy norm estimate

$$
\left|u-u^{h}\right|_{1,2, \Omega} \leq C_{0}\left(\sum_{K \in T_{h}} h_{K}^{2}|u|_{2,2, K}^{2}\right)^{1 / 2},
$$

where $|\cdot|_{m, 2, \omega}$ denotes the seminorm of highest-order derivatives in the Sobolev space $H^{m}(\omega)$. In case (1.5), we would use the above algorithm with $h_{K}$ in (1.3) and (1.4) replaced by $h_{K}^{2}$. The estimate $(0.2)$ would be used also in this case to prove that $D_{H}^{2}\left(u^{h} ; K\right)$ is a sufficiently good approximation to $|u|_{2, \infty, K}$; cf. the analysis 
below. In case $(1.6)$, the new mesh $\tilde{T}_{h}$ in $4^{\circ}$ would be constructed so that

$$
\begin{gathered}
h_{\tilde{K}}^{2} D_{H}^{2}\left(u^{h} ; K\right) \simeq \gamma=\text { constant }, \quad \forall \tilde{K} \in \tilde{T}_{h} \text { with } \tilde{K} \subseteq K, \\
C_{0}\left(\sum_{K \in \tilde{T}_{h}} \gamma^{2}\right)^{1 / 2}=C_{0} \sqrt{\tilde{N}} \gamma=\delta,
\end{gathered}
$$

where $\tilde{N}$ is the number of elements in $\tilde{T}_{h}$. Again, (0.2) would be used to justify the algorithm. Note that (1.5) and (1.7) give the same control up to the choice of the tolerance $\delta$. For numerical experiments with error control based on (1.5), see Section 4 below. For a further discussion, see [9].

Remark 1.3. In any adaptive method we face two problems, namely, (i) estimation of the error and check if the error is below the given tolerance, and (ii) construction of a properly refined new grid if the error is above the tolerance. It is not enough to solve just problem (i). Even if we can accurately estimate the error $e(x)$ for all $x \in \Omega$, it is not clear how to properly refine the mesh to decrease the error, if too large. In general, one should not refine everywhere where the error is too large since in an elliptic problem some effects are global, for instance, a corner singularity may cause a large error also away from the corner. To decrease the error in such a case, we should not refine everywhere but only close to the corner. A main difference between the Babuska approach and our approach is that we base the adaptive algorithm on an error estimate which exhibits the structure of the error and which is used to solve both problems (i) and (ii). It appears to us that in the Babuska approach it is less obvious how to solve (ii), and that this is the reason why this method is more difficult to justify theoretically.

\section{Analysis in a Model Case.}

2.1. The Exact Solution. We shall now analyze and justify the proposed adaptive algorithm under the assumption that the exact solution $u$ belongs to the Sobolev space $W_{\infty}^{\beta}(\Omega)$ and satisfies the following estimate: There are constants $c$ and $C$ such that for all $x \in \Omega$,

$$
\begin{gathered}
c|x|^{\beta-2} \leq D^{2} u(x) \leq C|x|^{\beta-2}, \\
\left|D^{\gamma} u(x)\right| \leq C|x|^{\beta-3} \text { for }|\gamma|=3,
\end{gathered}
$$

where

$$
D^{2} u(x)=\max \left\{\left|D^{\alpha} u(x)\right|:|\alpha|=2\right\},
$$

and $1<\beta<2$. Note that this corresponds to a situation where the exact solution $u(x)$ has a singularity at the origin of strength $|x|^{\beta}$. For simplicity we assume that the origin belongs to the interior of $\Omega$. A more realistic situation would be to consider the case of a singularity located at a corner of $\Omega$; a singularity of strength $|x|^{\beta}$ with $1<\beta<2$ would then correspond to a corner angle $\kappa=\pi / \beta$ satisfying $\pi / 2<\kappa<\pi$. Of course the restriction $\beta>1$ is related to the fact that we seek to control the quantity $\|\nabla u\|_{\infty, \Omega}$, which requires $\|\nabla u\|_{\infty, \Omega}$ to be bounded, whereas we assume $\beta<2$ to have a singularity of sufficient strength for a refinement to be necessary.

Observe that in applying the algorithm in the above case we do not, of course, use any a priori knowledge of the nature of the exact solution like (2.1). The only 
data required for the algorithm is $\Omega, f$ and $\delta$. What we prove is that if $(2.1)$ is satisfied, then the algorithm will perform as desired.

To simplify the presentation, we shall further assume that the region $\Omega$ is convex with smooth boundary $\Gamma$. The functions $v$ in the finite element space $V_{h}$ are assumed to be piecewise linear on the triangulation $T_{h}=\{K\}$, corresponding to a polygonal approximation $\Omega_{h}$ of $\Omega$, and extended by zero in $\Omega \backslash \Omega_{h}$.

2.2. Optimal Meshes. Let us now first see what a reasonable mesh would look like in the case (2.1), assuming that we want to satisfy $(0.3)$. It is then convenient to divide $\Omega$ into subregions $\Omega_{j}$ according to the size of the second derivatives of $u$. We thus introduce

$$
\Omega_{j}=\left\{x \in \Omega: 2^{-j}<|x| \leq 2^{-j+1}\right\}
$$

and we assume that $\bigcup_{j \geq 0} \Omega_{j}=\Omega$. We then have by (2.1)

$$
c d_{j}^{\beta-2} \leq|u|_{2, \infty, \Omega_{j}} \leq C d_{j}^{\beta-2},
$$

where $d_{j}=2^{-j}$. From (0.3) it follows that we should choose the mesh size $h_{j}$ in $\Omega_{j}$ so that $h_{j} d_{j}^{\beta-2} \sim \delta$, i.e.,

$$
h_{j} \sim \delta d_{j}^{2-\beta}
$$

as long as $h_{j} \leq d_{j}$, i.e., as long as $j \leq J$, where

$$
d_{J}^{\beta-1} \sim \delta .
$$

Here and below, we write $a \sim b$ if $c a \leq b \leq C a$. Further, we should choose the mesh size $\sim \delta^{1 /(\beta-1)}$ in $\Omega_{J}^{*}=\left\{x \in \Omega:|x| \leq 2^{-J}\right\}$. It is clear that the choice (2.3) is best possible, and we shall prove below that our algorithm will produce a final mesh satisfying (2.3).

2.3 Analysis of Step 1. We first recall the following optimal global maximum norm estimate (cf. [17]): For the finite element method (1.1) on the quasi-uniform mesh $T_{\bar{h}}$ with solution $u^{\bar{h}}$ we have for $0 \leq s \leq 1$

$$
\left\|\nabla\left(u-u^{\bar{h}}\right)\right\|_{\infty, \Omega} \leq C \bar{h}^{s}|u|_{s+1, \infty, \Omega},
$$

where $|\cdot|_{s, \infty, \Omega}$ denotes the norm in the usual Sobolev space $W_{\infty}^{s}(\Omega)$ of functions with derivatives of order $s$ in $L_{\infty}(\Omega)$. Let us now start the analysis of the algorithm in the special case of an exact solution $u \in W_{\infty}^{\beta}(\Omega)$ satisfying $(2.1)$. We then begin with the quasi-uniform partition $T_{\bar{h}}$ with elements of size $\bar{h}$ and the corresponding finite element solution $u^{\bar{h}}$. We shall use the following local version of the estimate (2.5) with $s=1$, which states that the finite element error in $\Omega_{j}$ is comparable to the interpolation error in $\Omega_{j}$, if $d_{j} / \bar{h}$ is large enough.

LEMMA 2.1. There are constants $C$ and $C_{1}$ such that, if $d_{j} \geq C_{1} \bar{h}$, then

$$
\left\|\nabla\left(u-u^{\bar{h}}\right)\right\|_{\infty, \Omega_{j}} \leq C \bar{h} d_{j}^{\beta-2} .
$$

Below we shall give an extension of Lemma 2.1 to more general meshes. This result, which is the key technical result we will need, involves a generalization of the earlier estimate (2.5) to non-quasi-uniform meshes and also, as indicated, a localization.

We postpone the proof of Lemma 2.1 and first show how this result can be used to prove that $D_{H}^{2} u^{\bar{h}}(x)$ will be a reasonable approximation of $D^{2} u(x)$ for $x \in \Omega_{j}$ if $H / \bar{h}$ and $d_{j} / \bar{h}$ are big enough. More precisely, we shall prove the following result. 
LEMMA 2.2. There are constants $c, C$ and $\bar{C}$ such that, if $H=C \bar{h}$, then

$$
\begin{gathered}
c \leq \frac{\left|D_{H}^{2} u^{\bar{h}}(x)\right|}{\left|D^{2} u(x)\right|} \leq C \quad \text { if }|x| \geq \overline{C h}, \\
c \bar{h}^{\beta-2} \leq\left|D_{H}^{2} u^{\bar{h}}(x)\right| \leq C \bar{h}^{\beta-2} \quad \text { if }|x| \leq \overline{C h} .
\end{gathered}
$$

Proof. We have by (2.1) and Lemma 2.1, if $x \in \Omega_{j}, d_{j} \geq 2 C_{1} \bar{h}, d_{j} \geq 2 H$, $|\alpha|=|\gamma|=1$,

$$
\begin{aligned}
\left|D_{H}^{\gamma} D^{\alpha} u^{\bar{h}}(x)-D^{\gamma+\alpha} u(x)\right| & \leq\left|D_{H}^{\gamma} D^{\alpha}\left(u^{\bar{h}}-u\right)(x)\right|+\left|\left(D_{H}^{\gamma}-D^{\gamma}\right) D^{\alpha} u(x)\right| \\
& \leq \frac{C \bar{h}}{H} d_{j}^{\beta-2}+C H d_{j}^{\beta-3}
\end{aligned}
$$

where we used a standard approximation result to estimate the second term. Now, if we here choose $H=C \bar{h}$ and $d_{j} \geq C H$ with $C$ sufficiently large, then it follows that

$$
\left|D_{H}^{\gamma} D^{\alpha} u^{\bar{h}}(x)-D^{\gamma+\alpha} u(x)\right| \leq \frac{1}{2} D^{2} u(x)
$$

if $x \in \Omega_{j}, d_{j} \geq \overline{C h}$ and $H=C \bar{h}$. Further, by the global estimate (2.5) with $s=\beta-1$ and an inverse estimate it also follows that there is a constant $C$ such that, if $H \geq \bar{h}$, then

$$
\left|D_{H}^{\gamma} D^{\alpha} u^{\bar{h}}(x)\right| \leq C \bar{h}^{\beta-2} .
$$

Recalling now the definition (1.2), we easily obtain the desired estimates $(2.7 \mathrm{a}, \mathrm{b})$ by combining (2.1), (2.8) and (2.9) (note that the constant $\bar{C}$ in (1.2) is assumed to be the same as the constant $\bar{C}$ in the lemma).

By Lemma 2.2 we may use $u^{\bar{h}}$ to accurately compute a local mesh size $h_{j}$ in $\Omega_{j}$ satisfying (2.3), if $d_{j} \geq \overline{C h}$, i.e., knowing $u^{\bar{h}}$, we may decide on a reasonable refinement in $\{|x| \geq \overline{C h}\}$ (for simplicity we write $\{|x| \leq d\}$ to mean $\{x \in \Omega:|x| \leq$ $d\})$. In the region $\{|x| \leq \overline{C h}\}$ we will, by $(2.7 \mathrm{~b})$, be led to a refinement with mesh size $c \delta \bar{h}^{2-\beta}$. Thus, if in stage $4^{\circ}$ of the algorithm the stopping criterion is not satisfied, a new mesh $T_{h}$ will be constructed which is a refinement of the first quasi-uniform mesh $T_{\bar{h}}$ and which will have the following characteristics (here $h(x)$ is a measure of the mesh size at $x$ ):

$$
\begin{gathered}
h(x) \sim \delta d_{j}^{2-\beta} \quad \text { if } x \in \Omega_{j}, d_{j} \geq \overline{C h}, \\
h(x) \sim \underline{h}:=\delta \bar{h}^{2-\beta} \quad \text { if }|x| \leq \overline{C h}
\end{gathered}
$$

where $\delta \leq C \bar{h}^{\beta-1}$; in fact, we have assumed that $\bar{h} \geq c \delta$ and $\bar{h} \leq C$. This means in particular that the mesh $T_{h}$ obtained after the first quasi-uniform mesh $T_{\bar{h}}$ will be correctly refined in $\{|x| \geq \overline{C h}\}$ and will be quasi-uniform in $\{|x| \leq \overline{C h}\}$ with typical mesh size $\underline{h} \sim \delta \bar{h}^{2-\beta}$.

2.4 Analysis of Step 2. We now continue the analysis starting with a mesh $T_{h}$ with local mesh size $h(x)$ satisfying (2.10). We shall in this case use the following generalizations of Lemmas 2.1 and 2.2, the difference being that the mesh is now no longer quasi-uniform. 
LEMMA 2.3. Suppose the mesh $T_{h}$ satisfies (2.10). Then there are constants $C$ and $C_{1}$ such that, if $d_{j} \geq C_{1} \underline{h}$, then

$$
\left\|\nabla\left(u-u^{h}\right)\right\|_{\infty, \Omega_{j}} \leq C h_{j} d_{j}^{\beta-2}
$$

where, according to (2.10), $h_{j}:=\max \left(\delta d_{j}^{2-\beta}, \delta \bar{h}^{2-\beta}\right)$ is the local mesh size on $\Omega_{j}$.

LEMMA 2.4. Suppose that the mesh $T_{h}$ satisfies (2.10). Then there are constants $c, C$ and $\bar{C}$ such that, if $x \in K \cap \Omega_{j}, d_{j} \geq \bar{C} \underline{h}$ and $H=C h_{K}$, then

$$
c \leq \frac{\left|D_{H}^{2} u^{h}(x)\right|}{\left|D^{2} u(x)\right|} \leq C ;
$$

further, if $|x| \leq \bar{C} \underline{h}$ and $H=C \underline{h}$, then

$$
c \underline{h}^{\beta-2} \leq\left|D_{H}^{2} u^{h}(x)\right| \leq C \underline{h}^{\beta-2} .
$$

The proof of Lemma 2.3 will be given below. The proof of Lemma 2.4 is analogous to the proof of Lemma 2.2 above and relies on Lemma 2.3 .

From Lemma 2.4 it follows that $D_{H}^{2} u^{h}(x)$ will be a reasonable approximation of $D^{2} u(x)$ in $\{|x| \geq \bar{C} \underline{h}\}$. Thus, if the stopping criterion is not satisfied, the algorithm will produce a refinement $\tilde{T}_{h}$ of $T_{h}$ with the following characteristics:

$$
\begin{array}{ll}
h(x) \sim \delta d_{j}^{2-\beta} & \text { if } x \in \Omega_{j}, d_{j} \geq \bar{C} \underline{h}, \\
h(x) \sim \underline{\underline{h}}:=\delta \underline{h}^{2-\beta} & \text { if }|x| \leq \bar{C} \underline{h},
\end{array}
$$

and $\delta \leq C \underline{h}^{\beta-1}$. Redefining now $T_{h}=\tilde{T}_{h}$ and letting $\bar{h}$ and $\underline{h}$ take the roles of $\underline{h}$ and $\underline{h}$, we then have again the same situation as at the start of Step 2 at the beginning of this subsection, i.e., a mesh $T_{h}$ satisfying $(2.10)$. The process may now be repeated.

2.5. The Number of Steps: Statement of Main Result. Let us now see how many steps will be required to obtain a mesh on which the error tolerance is satisfied. For simplicity, we start with a quasi-uniform mesh $T_{\bar{h}}$ with mesh length $\bar{h}=\delta$. We denote by $\underline{h}_{n}$ the minimal mesh length of the triangulation $T_{h}=T_{h}^{n}$ obtained after $n$ steps. According to $(2.10 \mathrm{~b})$, we will then have

$$
\underline{h}_{n}=\delta \underline{h}_{n-1}^{2-\beta}, \quad n \geq 1,
$$

where $\underline{h}_{0}=\bar{h}=\delta$, and consequently

$$
\begin{aligned}
\underline{h}_{n} & =\delta \underline{h}_{n-1}^{2-\beta}=\delta \delta^{(2-\beta)} \underline{h}_{n-2}^{(2-\beta)^{2}} \\
& =\delta \delta^{(2-\beta)} \cdots \delta^{(2-\beta)^{n}}=\delta^{\left(1-(2-\beta)^{n+1}\right) /(\beta-1)},
\end{aligned}
$$

so that

$$
\underline{h}_{n}^{\beta-1}=\delta^{1-(2-\beta)^{n+1}} .
$$

Now, as a by-product of the proof of Lemma 2.3, we have the following error estimate which gives a generalization of $(2.5)$ to a non-quasi-uniform mesh: If $T_{h}$ satisfies (2.10) then

$$
\left\|\nabla\left(u-u^{h}\right)\right\|_{\infty, \Omega} \leq C \underline{h}^{\beta-1} .
$$

In particular, we thus have, recalling (2.13),

$$
\left\|\nabla\left(u-u_{n}^{h}\right)\right\|_{\infty, \Omega} \leq C \underline{h}_{n}^{\beta-1}=C \delta^{1-(2-\beta)^{n+1}} .
$$


This means that we will not in a finite number of steps achieve $\left\|\nabla\left(u-u^{h}\right)\right\|_{\infty, \Omega} \leq$ $C \delta$. In practice, this does not of course pose a problem; we may, e.g., assume that $n$ is chosen so that

$$
\delta^{-(2-\beta)^{n+1}} \leq C
$$

with $C$ a moderate constant. The qualitative conclusion from (2.14) is that the required number of steps would (slowly) increase as $\beta$ approaches $1, \beta>1$.

We can now summarize our main result as follows:

THEOREM. Suppose the exact solution u satisfies (2.1). Then the adaptive algorithm with initial mesh length $\bar{h}=\delta$ will produce a sequence of meshes $T_{h}^{n}$, $n=0,1,2, \ldots$, with corresponding finite element solutions $u_{n}^{h}\left(u_{0}^{h}=u^{\bar{h}}\right)$, such that

$$
\left\|\nabla\left(u-u_{n}^{h}\right)\right\|_{\infty, \Omega} \leq C \delta^{1-(2-\beta)^{n+1}} .
$$

Further, the mesh $T_{h}^{n}$ will be correctly refined in the region $\left\{|x| \geq \bar{C} \underline{h}_{n-1}\right\}$ where $\underline{h}_{n-1}$, the minimal size of elements in $T_{h}^{n-1}$, is given by

$$
\underline{h}_{n-1}=\delta^{\theta_{n}}, \quad \theta_{n}=\left(1-(2-\beta)^{n}\right) /(\beta-1),
$$

and the region $\left\{|x| \leq \bar{C} \underline{h}_{n-1}\right\}$ will have a quasi-uniform mesh of size $\underline{h}_{n}$.

3. Proofs of Lemmas 2.1 and 2.3. It remains to prove the local estimate (2.11) of Lemma 2.3. Clearly Lemma 2.1 can be viewed as a special case of Lemma 2.3 and does not require a separate proof. For the proof of Lemma 2.3 we fix $j$ and an arbitrary point $x_{0} \in \Omega_{j}$, together with the element $K_{0}$ containing $x_{0}$, for which we may assume $\operatorname{dist}\left(K_{0}, 0\right) \sim d_{j}$. We let $\tilde{u}^{h} \in V_{h}$ denote the piecewise linear interpolant of $u$ and write $\partial_{i}:=\partial / \partial x_{i}, i=1,2$.

For the interpolant $\tilde{u}^{h}$ we have

$$
\left\|\nabla\left(u-\tilde{u}^{h}\right)\right\|_{\infty, K_{0}} \leq C h_{K_{0}}|u|_{2, \infty, K_{0}} \leq C h_{j} d_{j}^{\beta-2},
$$

and hence, by the triangle inequality,

$$
\left|\partial_{i}\left(u-u^{h}\right)\left(x_{0}\right)\right| \leq C h_{j} d_{j}^{\beta-2}+\left|\partial_{i}\left(\tilde{u}^{h}-u^{h}\right)\left(x_{0}\right)\right| .
$$

Since $\partial_{i}\left(\tilde{u}^{h}-u^{h}\right)$ is constant on $K_{0}$, the last term can be represented as

$$
\left|\partial_{i}\left(\tilde{u}^{h}-u^{h}\right)\left(x_{0}\right)\right|=\left|\left(\partial_{i}\left(\tilde{u}^{h}-u^{h}\right), \delta_{0}\right)\right|,
$$

where $\delta_{0}$ is a smooth approximate delta function, supported in $K_{0}$, and such that

$$
h_{K_{0}}^{2}\left\|\delta_{0}\right\|_{\infty, K_{0}} \leq C \text {. }
$$

Thus,

$$
\begin{aligned}
\left|\partial_{i}\left(u-u^{h}\right)\left(x_{0}\right)\right| & \leq C h_{j} d_{j}^{\beta-2}+\left|\left(\partial_{i}\left(\tilde{u}^{h}-u^{h}\right), \delta_{0}\right)\right| \\
& \leq C h_{j} d_{j}^{\beta-2}+\left|\left(\partial_{i}\left(u-u^{h}\right), \delta_{0}\right)\right|
\end{aligned}
$$

where we have again used the triangle inequality. Let now $G$ solve the adjoint problem

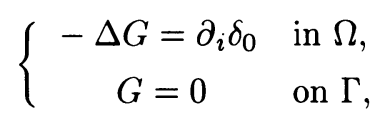

and let $G^{h} \in V_{h}$ be the Ritz projection of $G$ determined by

$$
a\left(v, G-G^{h}\right)=0 \quad \forall v \in V_{h} .
$$


Then,

$$
\begin{aligned}
\left(\partial_{i}\left(u-u^{h}\right), \delta_{0}\right) & =-\left(u-u^{h}, \partial_{i} \delta_{0}\right)=-\left(u-u^{h},-\Delta G\right) \\
& =-a\left(u-u^{h}, G\right)=a\left(u-u^{h}, G^{h}-G\right)=a\left(u-\tilde{u}^{h}, G^{h}-G\right) .
\end{aligned}
$$

Below we shall show, with $e:=G^{h}-G$, that

$$
\|\nabla e\|_{L_{1}(\Omega)} \leq C
$$

and

$$
\|\nabla e\|_{\Omega_{j+2}^{*}} \leq C d_{j}^{-1}
$$

where $\|\cdot\|_{\omega}$ denotes the $L_{2}(\omega)$-norm and $\Omega_{k}^{*}=\left\{x \in \Omega:|x| \leq 2^{-k}\right\}$ as above. Using (3.1), (3.2) and (3.3), together with approximation properties of the interpolant $\tilde{u}^{h}$, we find

$$
\begin{aligned}
& \left|\left(\partial_{i}\left(u-u^{h}\right), \delta_{0}\right)\right|=\left|a\left(u-\tilde{u}^{h}, e\right)\right| \\
& \quad \leq \sum_{k \leq j+2}\left\|\nabla\left(u-\tilde{u}^{h}\right)\right\|_{\infty, \Omega_{k}}\|\nabla e\|_{L_{1}\left(\Omega_{k}\right)}+C \sum_{k>j+2}\left\|\nabla\left(u-\tilde{u}^{h}\right)\right\|_{\infty, \Omega_{k}} d_{k}\|\nabla e\|_{\Omega_{k}} \\
& \quad \leq \max _{k \leq j+2}\left\|\nabla\left(u-\tilde{u}^{h}\right)\right\|_{\infty, \Omega_{k}}\|\nabla e\|_{L_{1}(\Omega)} \\
& \quad+C\left(\sum_{k>j+2}\left\|\nabla\left(u-\tilde{u}^{h}\right)\right\|_{\infty, \Omega_{k}}^{2} d_{k}^{2}\right)^{1 / 2}\|\nabla e\|_{\Omega_{j+2}^{*}} \\
& \quad \leq C \max _{k \leq j+2} h_{k} d_{k}^{\beta-2}+C\left(\sum_{k>j+2} h_{k}^{2} d_{k}^{2 \beta-2}\right)^{1 / 2} d_{j}^{-1} \\
& \quad \leq C h_{j} d_{j}^{\beta-2},
\end{aligned}
$$

where in the last step we have used the definitions of $d_{k}$ and $h_{k}$ to get

$$
\begin{aligned}
\max _{k \leq j+2} h_{k} d_{k}^{\beta-2} & =\max _{k \leq j+2} \max \left(\delta \bar{h}^{2-\beta} d_{k}^{\beta-2}, \delta\right) \\
& =\max \left(\delta \bar{h}^{2-\beta} d_{j+2}^{\beta-2}, \delta\right)=h_{j+2} d_{j+2}^{\beta-2} \leq C h_{j} d_{j}^{\beta-2}
\end{aligned}
$$

and

$$
\begin{aligned}
\sum_{k>j+2} h_{k}^{2} d_{k}^{2 \beta-2} & =\sum_{k>j+2} \delta^{2} \max \left(\bar{h}^{4-2 \beta} d_{k}^{2 \beta-2}, d_{k}^{2}\right) \\
& \leq 2 \delta^{2} \max \left(\bar{h}^{4-2 \beta} \sum_{k>j+2} d_{k}^{2 \beta-2}, \sum_{k>j+2} d_{k}^{2}\right) \\
& \leq C \delta^{2} \max \left(\bar{h}^{4-2 \beta} d_{j}^{2 \beta-2}, d_{j}^{2}\right)=C h_{j}^{2} d_{j}^{2 \beta-2}
\end{aligned}
$$

In order to prove Lemma 2.3 , it now suffices to verify (3.2) and (3.3). For this purpose we introduce the domains (cf. Figure 3.1)

$$
\omega_{k}=\left\{x \in \Omega: 2^{-k}<\left|x-x_{0}\right| \leq 2^{-k+1}\right\}
$$

and

$$
\omega_{k}^{*}=\left\{x \in \Omega:\left|x-x_{0}\right| \leq 2^{-k}\right\}
$$


For the proof of (3.2) we have first

$$
\|\nabla e\|_{L_{1}(\Omega)}=\sum_{k \geq J}\|\nabla e\|_{L_{1}\left(\omega_{k}\right)}+\|\nabla e\|_{L_{1}\left(\omega_{J}^{*}\right)} \leq C S+C d_{J}\|\nabla e\|_{\omega_{J}^{*}},
$$

where

$$
S:=\sum_{k \leq J} d_{k}\|\nabla e\|_{\omega_{k}}
$$

and where $J$ will be determined later. For the moment we only assume that $J$ is not too big so that whenever $k \leq J$ and $K$ intersects $\omega_{k}, K \in T_{h}$, then $K \subseteq \omega_{k}^{\prime}:=$ $\omega_{k-1} \cup \omega_{k} \cup \omega_{k+1}$. We shall prove below that for a suitable choice of such a $J$, we have

$$
S \leq C+C d_{J}\|\nabla e\|_{\omega_{J}^{*}}+\frac{1}{2} S
$$

and

$$
d_{J}\|\nabla e\|_{\omega_{J}^{*}} \leq C
$$

Together, these estimates will complete the proof of (3.2). For the proofs of (3.4) and (3.3), we shall need the following local error estimate.

LEMMA 2.5. There is a constant $C$ such that the following holds: If $\omega \subseteq \omega^{\prime} \subseteq \Omega$, $d:=\operatorname{dist}\left(\omega, \Omega \backslash \omega^{\prime}\right)>0$, and $\operatorname{diam}(K) \leq \frac{1}{2} d$ whenever $K$ intersects $\omega, K \in T_{h}$, then

$$
\|\nabla e\|_{\omega} \leq C \inf _{\chi \in V_{h}}\left(\|\nabla(G-\chi)\|_{\omega^{\prime}}+d^{-1}\|G-\chi\|_{\omega^{\prime}}\right)+C d^{-1}\|e\|_{\omega^{\prime}} .
$$

Proof. Let $\chi \in V_{h}$ be arbitrary and put $\zeta=G^{h}-\chi$ and $\eta=\chi-G$, and let $\varphi$ be a smooth cutoff function such that $\varphi=1$ on $\omega, \varphi=0$ on each $K$ intersecting $\Omega \backslash \omega^{\prime}$, and

$$
|\varphi|_{l, \infty} \leq C d^{-l} \text { for } l=0,1,2 .
$$

Using integration by parts and the error equation

$$
a(v, e)=(\nabla v, \nabla e)=0 \quad \forall v \in V_{h},
$$

we find that, with $\|\cdot\|=\|\cdot\|_{\Omega}$,

$$
\begin{aligned}
\|\varphi \nabla \varsigma\|^{2} & =\left(\nabla\left(\varphi^{2} \varsigma\right), \nabla \varsigma\right)-2(\varsigma \nabla \varphi, \varphi \nabla \varsigma) \\
& =\left(\nabla\left(\varphi^{2} \varsigma\right), \nabla e\right)-\left(\nabla\left(\varphi^{2} \varsigma\right), \nabla \eta\right)-2(\varsigma \nabla \varphi, \varphi \nabla \varsigma) \\
& =\left(\nabla\left(\varphi^{2} \varsigma-\left(\varphi^{2} \varsigma\right)^{h}\right), \nabla e\right)-\left(\nabla\left(\varphi^{2} \varsigma\right), \nabla \eta\right)-2(\varsigma \nabla \varphi, \varphi \nabla \zeta) \\
& =\left(\nabla\left(\varphi^{2} \varsigma-\left(\varphi^{2} \varsigma\right)^{h}\right), \nabla \zeta\right)-\left(\nabla\left(\varphi^{2} \varsigma\right)^{h}, \nabla \eta\right)-2(\varsigma \nabla \varphi, \varphi \nabla \varsigma),
\end{aligned}
$$

where $\left(\varphi^{2} \varsigma\right)^{h} \in V_{h}$ denotes the piecewise linear interpolant of $\varphi^{2} \varsigma$ satisfying

$$
\begin{gathered}
\left\|\nabla\left(\varphi^{2} \varsigma\right)^{h}\right\| \leq C\left\|\nabla\left(\varphi^{2} \varsigma\right)\right\|, \\
\left\|\nabla\left(\varphi^{2} \varsigma-\left(\varphi^{2} \varsigma\right)^{h}\right)\right\|_{K} \leq C h_{K} \sum_{|\alpha|=2}\left\|D^{\alpha}\left(\varphi^{2} \varsigma\right)\right\|_{K} \quad \forall K \in T_{h} .
\end{gathered}
$$

We shall now estimate the three terms on the right-hand side of (3.7). By (3.9) and (3.6), together with the fact that $\varsigma$ is piecewise linear, and using an inverse 
estimate on each $K$, we obtain

$$
\begin{aligned}
& \left|\left(\nabla\left(\varphi^{2} \varsigma-\left(\varphi^{2} \varsigma\right)^{h}\right), \nabla \zeta\right)\right| \\
& \leq C \sum_{\substack{K \\
K \subseteq \omega^{\prime}}} h_{K}\left(d^{-2}\|\zeta\|_{K}+d^{-1}\|\varphi \nabla \varsigma\|_{K}\right)\|\nabla \zeta\|_{K} \\
& \leq C \sum_{\substack{K \\
K \subseteq \omega^{\prime}}}\left(d^{-1}\left\|_{\zeta}\right\|_{K}+\|\varphi \nabla \zeta\|_{K}\right) d^{-1}\left\|_{\zeta}\right\|_{K} \\
& \leq C d^{-2}\|\zeta\|_{\omega^{\prime}}^{2}+\frac{1}{4}\|\varphi \nabla \zeta\|^{2} .
\end{aligned}
$$

Using (3.8), we find that

$$
\begin{aligned}
& \left|\left(\nabla\left(\varphi^{2} \varsigma\right)^{h}, \nabla \eta\right)\right| \leq C\left\|\nabla\left(\varphi^{2} \varsigma\right)\right\|\|\nabla \eta\|_{\omega^{\prime}} \\
& \quad \leq C d^{-2}\|\varsigma\|_{\omega^{\prime}}^{2}+\frac{1}{4}\|\varphi \nabla \zeta\|^{2}+C\|\nabla \eta\|_{\omega^{\prime}}^{2} .
\end{aligned}
$$

Finally,

$$
|2(\varsigma \nabla \varphi, \varphi \nabla \varsigma)| \leq C d^{-2}\|\zeta\|_{\omega^{\prime}}^{2}+\frac{1}{4}\|\varphi \nabla \varsigma\|^{2} .
$$

Altogether we now have

$$
\|\varphi \nabla \varsigma\|^{2} \leq C\left(d^{-2}\|\varsigma\|_{\omega^{\prime}}^{2}+\|\nabla \eta\|_{\omega^{\prime}}^{2}\right)+\frac{3}{4}\|\varphi \nabla \varsigma\|^{2},
$$

and hence

$$
\|\varphi \nabla \varsigma\| \leq C\left(d^{-1}\|\varsigma\|_{\omega^{\prime}}+\|\nabla \eta\|_{\omega^{\prime}}\right) .
$$

For the error $e$, this implies

$$
\begin{aligned}
\|\nabla e\|_{\omega} & \leq\|\varphi \nabla \zeta\|+\|\nabla \eta\|_{\omega} \leq C\left(d^{-1}\|\zeta\|_{\omega^{\prime}}+\|\nabla \eta\|_{\omega^{\prime}}\right) \\
& \leq C\left(d^{-1}\|\eta\|_{\omega^{\prime}}+d^{-1}\|e\|_{\omega^{\prime}}+\|\nabla \eta\|_{\omega^{\prime}}\right)
\end{aligned}
$$

which completes the proof of Lemma 2.5.

We now continue with the proof of Lemma 2.3 with the purpose of first showing (3.4) and (3.5), and then (3.3). Applying Lemma 2.5 with $\omega=\omega_{k}$ and $\omega^{\prime}=\omega_{k}^{\prime}$, $\chi=\tilde{G}^{h}$ the piecewise linear interpolant of $G$, and

$$
H_{k}:=\delta \max \left(\bar{h}^{2-\beta},\left(\left|x_{0}\right|+d_{k}\right)^{2-\beta}\right) \sim \max _{K \subseteq \omega_{k}^{\prime}} h_{K}
$$

(cf. Fig 3.1), we obtain

$$
\begin{aligned}
S & =\sum_{k \leq J} d_{k}\|\nabla e\|_{\omega_{k}} \leq C \sum_{k \leq J+1}\left(d_{k}\left\|\nabla\left(G-\tilde{G}^{h}\right)\right\|_{\omega_{k}}+\left\|G-\tilde{G}^{h}\right\|_{\omega_{k}}+\|e\|_{\omega_{k}}\right) \\
& \leq C \sum_{k \leq J+2}\left(d_{k} H_{k}+H_{k}^{2}\right)|G|_{2,2, \omega_{k}}+C \sum_{k \leq J+1}\|e\|_{\omega_{k}} .
\end{aligned}
$$

In order to estimate $|G|_{2,2, \omega_{k}}$, we use the representation

$$
\begin{aligned}
D^{\alpha} G(x) & =\int_{\Omega} D_{x}^{\alpha} g(x, y) \partial_{i} \delta_{0}(y) d y \\
& =-\int_{K_{0}} \partial_{i} D_{x}^{\alpha} g(x, y) \delta_{0}(y) d y, \quad x \notin K_{0},
\end{aligned}
$$

where $g(x, y)$ is the associated Green's function, known to satisfy

$$
\left|D_{y}^{\gamma} D_{x}^{\alpha} g(x, y)\right| \leq C|x-y|^{-2-|\gamma|} \text { for }|\alpha|=2,|\gamma|=0,1 .
$$


Hence,

$$
|G|_{2,2, \omega_{k}} \leq C d_{k} \max _{|\alpha|=2} \max _{x \in \omega_{k}}\left|D^{\alpha} G(x)\right| \leq C d_{k}^{-2}\left\|\delta_{0}\right\|_{L_{1}\left(K_{0}\right)} \leq C d_{k}^{-2}
$$

and so

$$
\begin{gathered}
\sum_{k \leq J+2}\left(d_{k} H_{k}+H_{k}^{2}\right)|G|_{2,2, \omega_{k}} \leq C \sum_{k \leq J+2}\left(d_{k}^{-1} H_{k}+d_{k}^{-2} H_{k}^{2}\right) \\
\leq C\left(1+\max _{k \leq J} d_{k}^{-1} H_{k}\right) \sum_{k \leq J} d_{k}^{-1} H_{k},
\end{gathered}
$$

where in the last step we have used the facts that $d_{k}^{-1} \leq 4 d_{k-2}^{-1}$ and $H_{k} \leq H_{k-2}$. Below we shall choose $J$ in such a way that

$$
d_{J}^{-1} H_{J}=\max _{k \leq J} d_{k}^{-1} H_{k} \leq C .
$$

Further,

$$
\begin{aligned}
\sum_{k \leq J} & d_{k}^{-1} H_{k}=\sum_{k \leq J} \delta \max \left(\bar{h}^{2-\beta} d_{k}^{-1},\left(\left|x_{0}\right|+d_{k}\right)^{2-\beta} d_{k}^{-1}\right) \\
& \leq C \delta \max \left(\bar{h}^{2-\beta} \sum_{k \leq J} d_{k}^{-1},\left|x_{0}\right|^{2-\beta} \sum_{k \leq J} d_{k}^{-1}, \sum_{k \leq J} d_{k}^{1-\beta}\right) \\
& \leq C \delta \max \left(\bar{h}^{2-\beta} d_{J}^{-1},\left|x_{0}\right|^{2-\beta} d_{J}^{-1}, d_{J}^{1-\beta}\right) \\
& \leq C \delta \max \left(\bar{h}^{2-\beta} d_{J}^{-1},\left(\left|x_{0}\right|+d_{J}\right)^{2-\beta} d_{J}^{-1}\right) \\
& =C H_{J} d_{J}^{-1} .
\end{aligned}
$$

Hence we conclude that

$$
S \leq C+\sum_{k \leq J+1}\|e\|_{\omega_{k}}
$$

Let us now estimate $\|e\|_{\omega_{k}}$. We have that

$$
\|e\|_{\omega_{k}}=\sup (\varphi, e)
$$

where the supremum is taken over all $\varphi$ with support in $\omega_{k}$ and with $\|\varphi\|_{\omega_{k}}=1$. For such a $\varphi$, let $\phi$ be the solution of the adjoint problem

$$
\begin{aligned}
& -\Delta \phi=\varphi \quad \text { in } \Omega, \\
& \phi=0 \quad \text { on } \Gamma \text {, }
\end{aligned}
$$

and let $\tilde{\phi}^{h} \in V_{h}$ be the piecewise linear interpolant of $\phi$, so that

$$
\begin{aligned}
|(\varphi, e)| & =|a(\phi, e)|=\left|a\left(\phi-\tilde{\phi}^{h}, e\right)\right| \\
& \leq \sum_{l \leq J}\left\|\nabla\left(\phi-\tilde{\phi}^{h}\right)\right\|_{\omega_{l}}\|\nabla e\|_{\omega_{l}}+\left\|\nabla\left(\phi-\tilde{\phi}^{h}\right)\right\|_{\omega_{J}^{*}}\|\nabla e\|_{\omega_{J}^{*}} \\
& \leq C \sum_{l \leq J} H_{l}|\phi|_{2,2, \omega_{l}^{\prime}}\|\nabla e\|_{\omega_{l}}+C H_{J}|\phi|_{2,2, \omega_{J-1}^{*}}\|\nabla e\|_{\omega_{J}^{*}} .
\end{aligned}
$$

Here,

$$
|\phi|_{2,2, \omega_{l}} \leq C . d_{l} \min \left(d_{l}^{-2}, d_{k}^{-2}\right) d_{k}
$$


For $l \leq k-2$ and $l \geq k+2$, this follows from the representation

$$
D^{\alpha} \phi(x)=\int D_{x}^{\alpha} g(x, y) \varphi(y) d s
$$

using (3.10), and for $k-1 \leq l \leq k+1$, it is a consequence of the standard elliptic regularity estimate

$$
|\phi|_{2,2, \Omega} \leq C\|\varphi\| \text {. }
$$

Estimating $|\phi|_{2,2, \omega_{j-1}^{*}}$ by similar arguments, we find that

$$
\|e\|_{\omega_{k}} \leq C \sum_{l \leq J} H_{l} d_{l} \min \left(d_{l}^{-2}, d_{k}^{-2}\right) d_{k}\|\nabla e\|_{\omega_{l}}+C H_{J} d_{J} d_{k}^{-1}\|\nabla e\|_{\omega_{J}^{*}},
$$

and by changing order of summation we conclude

$$
\begin{aligned}
\sum_{k \leq J+1}\|e\|_{\omega_{k}} \leq & C \sum_{l \leq J}\|\nabla e\|_{\omega_{l}} H_{l} d_{l} \sum_{k \leq J+1} d_{k} \min \left(d_{l}^{-2}, d_{k}^{-2}\right) \\
& +C H_{J} d_{J}\|\nabla e\|_{\omega_{J}^{*}} \sum_{k \leq J+1} d_{k}^{-1} \\
\leq & C \sum_{l \leq J} H_{l}\|\nabla e\|_{\omega_{l}}+C H_{J}\|\nabla e\|_{\omega_{J}^{*}} \\
\leq & C_{*} \max _{l \leq J}\left(H_{l} d_{l}^{-1}\right) S+C H_{J}\|\nabla e\|_{\omega_{J}^{*}}
\end{aligned}
$$

In order to complete the proof of (3.4) we now choose $J$ such that

$$
\frac{1}{4 C_{*}}<H_{J} d_{J}^{-1}=\max _{l \leq J} H_{l} d_{l}^{-1} \leq \frac{1}{2 C_{*}} .
$$

It remains to show that (3.5) holds for this choice of $J$. We then note that by stability we have $\|\nabla e\| \leq\|\nabla G\|$, where

$$
\|\nabla G\|^{2}=a(G, G)=\left(G, \partial_{i} \delta_{0}\right)=-\left(\partial_{i} G, \delta_{0}\right) \leq\|\nabla G\|\left\|\delta_{0}\right\|_{K_{0}} .
$$

Together, these estimates show that

$$
d_{J}\|\nabla e\| \leq d_{J}\|\nabla G\| \leq d_{J}\left\|\delta_{0}\right\|_{K_{0}} \leq C d_{J} h_{K_{0}}^{-1} \leq C,
$$

where, in the last step, we have used that

$$
d_{J} \sim C_{*} H_{J} \leq C \delta \max \left(\bar{h}^{2-\beta},\left|x_{0}\right|^{2-\beta}, d_{J}^{2-\beta}\right),
$$

implying, since $\delta \leq C \bar{h}^{\beta-1}$, that

$$
d_{J} \leq C \delta \max \left(\bar{h}^{2-\beta},\left|x_{0}\right|^{2-\beta}\right) \sim h_{K_{0}} .
$$

It now remains to prove (3.3). For this we first note that as a part of the proof of (3.4) we have shown that

$$
\sum_{k \leq J+1}\|e\|_{\omega_{k}} \leq C
$$

which in turn implies that

$$
\|e\|_{\Omega_{j+1}^{*}} \leq C
$$




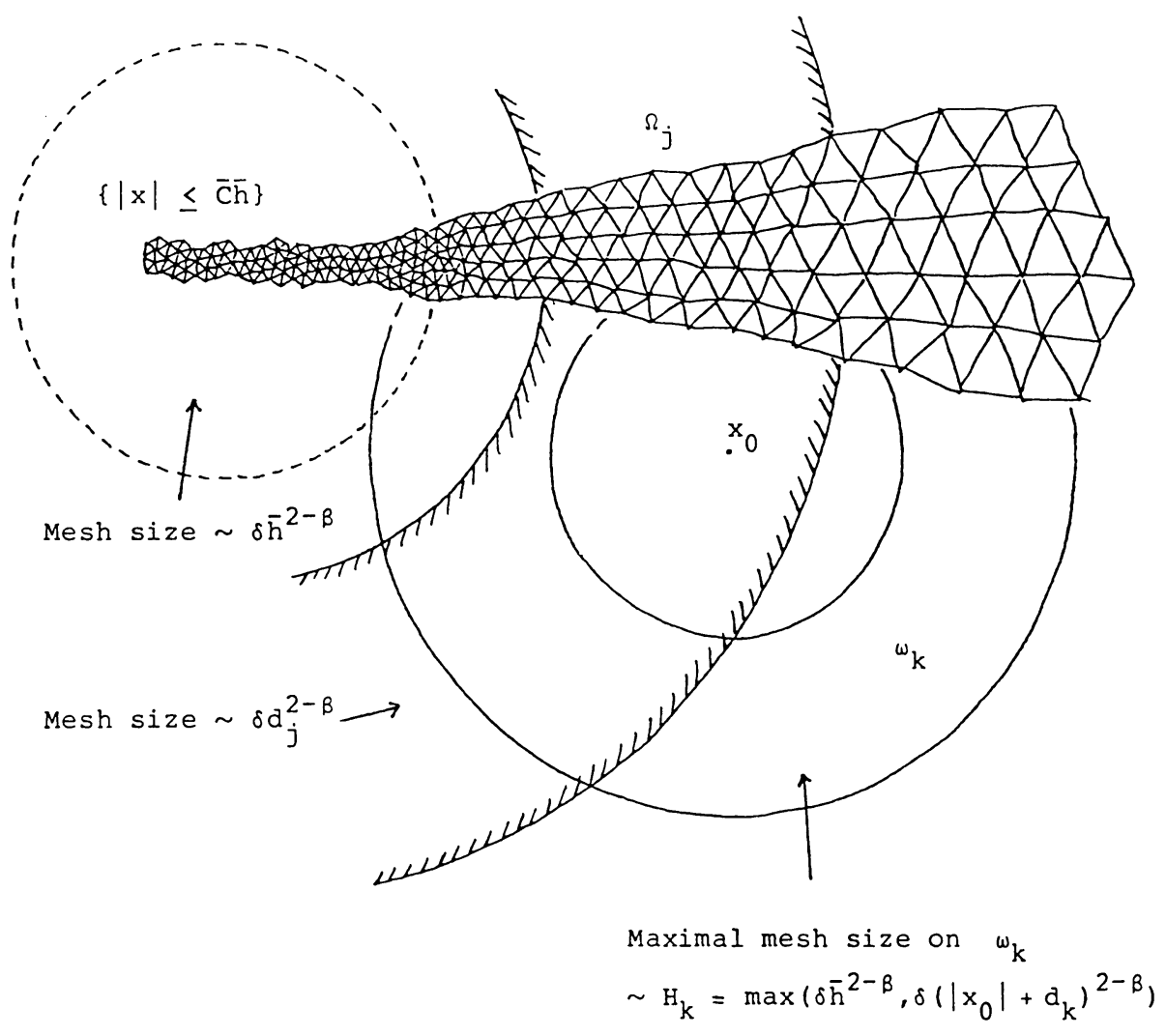

FIGURE 3.1

Mesh size depending on the distance to the origin.

To see this, note that the domain $\Omega_{j+1}^{*}$, provided that $d_{j} \geq C \underline{h}$ for a sufficiently large $C$, is covered by, say, at most two of the domains $\omega_{k}$.

Now let $\varphi$ be a cutoff function such that $\varphi=1$ on $\Omega_{j+2}^{*}, \varphi=0$ on each $K$ intersecting $\Omega \backslash \Omega_{j+1}^{*}$, and such that

$$
|\varphi|_{l, \infty} \leq C d_{j}^{-l} \text { for } l=0,1,2 .
$$

Then, by Lemma 2.5 with $\omega=\Omega_{j+2}^{*}, \omega^{\prime}=\Omega_{j+1}^{*}, d=d_{j} / 4$, and $\chi \equiv 0$, we have

$$
\|\nabla e\|_{\Omega_{j+2}^{*}} \leq C\left(\|\nabla G\|_{\Omega_{j+1}^{*}}+d_{j}^{-1}\|G\|_{\Omega_{j+1}^{*}}\right)+C d_{j}^{-1}\|e\|_{\Omega_{j+1}^{*}} \leq C d_{j}^{-1},
$$

where we have used our above estimate of $\|e\|_{\Omega_{j+1}^{*}}$ and the fact that

$$
\begin{aligned}
d_{j}\|\nabla G\|_{\Omega_{j+1}^{*}}+\|G\|_{\Omega_{j+1}^{*}} & \leq C\left(d_{j}^{2}\|\nabla G\|_{\infty, \Omega_{j+1}^{*}}+d_{j}\|G\|_{\infty, \Omega_{j+1}^{*}}\right) \\
& \leq C\left\|\delta_{0}\right\|_{L_{1}\left(K_{0}\right)} \leq C .
\end{aligned}
$$

This completes the proof of (3.3) and hence of Lemma 2.3.

Remark 3.1. From the error representation formula (3.1) it follows that the constant $C_{0}$ in (0.2) can be estimated as follows:

$$
C_{0} \leq\left(C_{1}+1\right) C_{2},
$$

where

$$
C_{1}=\max _{x_{0} \in \Omega}\left\|\nabla G^{h}\left(x_{0}, \cdot\right)\right\|_{L_{1}(\Omega)},
$$


with $G^{h}\left(x_{0}, \cdot\right)$ the discrete Green's function with pole at $x_{0} \in \Omega$ occurring in (3.1), and where $C_{2}$ is the error constant in interpolation with piecewise linear functions:

$$
\left|u-\tilde{u}^{h}\right|_{1, \infty, \Omega} \leq C_{2} \max _{K \in T_{h}} h_{K}|u|_{2, \infty, K} .
$$

The constant $C_{2}$ depends on the minimal angle of the triangulation $T_{h}$ and may easily be estimated (on reasonable triangulations one can probably take $C_{2} \sim 2$ in practice). Note that $C_{1}$ essentially depends only on $\Omega$ (and the coefficients in a variable coefficient generalization of $(0.1))$ and not on the right-hand side $f$. To compute $C_{1}$ approximately, it may in many cases be sufficient to compute $G^{h}\left(x_{0}, \cdot\right)$ on a coarse mesh for only a few conveniently chosen points $x_{0} \in \Omega$ (see [9]).

4. Numerical Results. In this section we give the results of some numerical experiments with the following variant of the algorithm analyzed above, where $3^{\circ}$ and $4^{\circ}$ are replaced by:

$3^{\circ \prime}$. For each $K \in T_{h}$ find $\nabla u^{h}\left(P_{K}\right)$ at the center of gravity $P_{K}$ of $K$ and also $\nabla u^{h}\left(P_{K^{\prime}}\right)$ for the set $N(K)$ of neighboring triangles $K^{\prime} \in T_{h}$ with one side in common with $K$ (see Figure 4.1) and set

$$
D_{H}^{2}\left(u^{h} ; K\right)=\max _{\substack{|\alpha|=1 \\ K^{\prime} \in N(K)}} \frac{\left|D^{\alpha} u^{h}\left(P_{K}\right)-D^{\alpha} u^{h}\left(P_{K^{\prime}}\right)\right|}{\left|P_{K}-P_{K^{\prime}}\right|}
$$

$4^{\circ \prime}$. If for all $K \in T_{h}$

$$
h_{K} D_{H}^{2}\left(u^{h} ; K\right) \leq \delta,
$$

then stop and accept the finite element solution $u^{h}$. Otherwise, construct a new mesh $\tilde{T}_{h}$ by repeatedly subdividing each triangle into four equal triangles until

$$
h_{\tilde{K}} D_{H}^{2}\left(u^{h} ; K\right) \leq \delta \quad \forall \tilde{K} \in \tilde{T}_{h} \text { with } \tilde{K} \subseteq K
$$

This algorithm essentially corresponds to the algorithm of Section 1 with $C_{0}=1$, $\bar{C}=0$ and $H=h_{K}$.

To implement the algorithm, we used the PLTMG-code by R. E. Bank [5] and simply replaced the Babuska type adaptivity, originally present in this code, by our own adaptivity. We kept the following feature of the original PLTMG-code: Successive meshes $T_{h}$ are chosen so that the number of degrees of freedom increases by approximately a factor of 4 . This means that in $4^{\circ \prime}$ the repeated subdivisions are only carried out as long as this condition is met. As a result, a somewhat larger number of steps than theoretically necessary is sometimes taken in practice. Notice also that in the PLTMG-code 'transition elements' obtained by subdivision into two triangles (obtained by introducing the dotted lines in Figure 4.2) are used to connect triangles with different subdivisions.

Such dotted lines are removed before and reintroduced after continued subdivisions, which means that no triangles with small angles will be constructed during the refinement process unless such triangles were present in the original quasi-uniform triangulation.

Example 4.1. We first give the results obtained for the problem

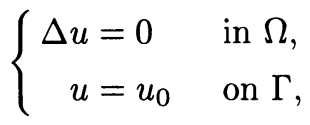




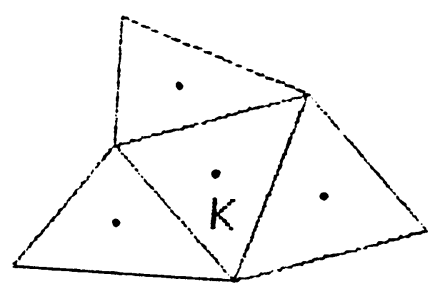

FIGURE 4.1

Evaluation points for computation of $D_{H}^{2}\left(u^{h} ; K\right)$.

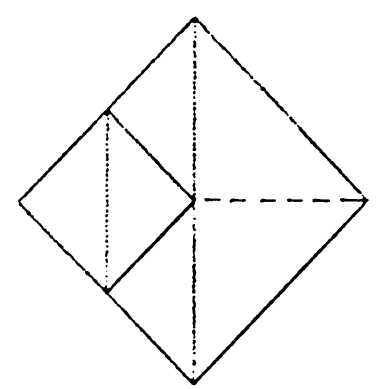

FiguRE 4.2

for the convex pie-shaped domain $\Omega=\{x=r(\cos \theta, \sin \theta): 0<r<1,0<\theta<$ $3 \pi / 4\}$ with exact solution $u(x)=r^{4 / 3} \sin 4 \theta / 3$. In Figure 4.3 we give the sequence of meshes produced by the algorithm with gradient control according to (4.1) with $\delta=.1$. In Figure 4.4 the actual gradient error $|\nabla e(x)|$ and error $|e(x)|$ are given as functions of the distance $|x|$ to the origin along the radius $\theta=\pi / 2$. We observe that the gradient error is very well controlled, and thus the algorithm performs as desired. We also note that the error $|e(x)|$ decreases as $x$ approaches the origin, as is to be expected, since the mesh is overly refined with respect to control of $\|e\|_{\infty, \Omega}$; cf. Example 4.2 below.

Example 4.2. To further illustrate the performance of the algorithm, we consider the following mixed Dirichlet-Neumann problem

$$
\left\{\begin{aligned}
\Delta u & =0 & & \text { in } \Omega \\
u & =u_{0} & & \text { on } \Gamma_{1}^{+} \cup \Gamma_{2} \\
\frac{\partial u}{\partial n} & =0 & & \text { on } \Gamma_{1}^{-}
\end{aligned}\right.
$$

with exact solution $u(x)=r^{1 / 2} \sin \theta / 2$, where $\Omega$ is the semidisc $\left\{x:|x| \leq 1, x_{2} \geq 0\right\}$ with boundary $\Gamma$ subdivided as follows: $\Gamma_{1}^{ \pm}=\left\{x \in \Gamma: x_{1} \gtrless 0, x_{2}=0\right\}, \Gamma_{2}=\{x \in$ $\left.\Gamma: x_{2}>0\right\}$. In this case, $u$ has a singularity of the form $r^{\beta}$ with $\beta<1$, so that $\nabla u(x)$ is singular and $|\nabla e(x)| \rightarrow \infty$ as $|x| \rightarrow 0$ on any mesh. 
378

KENNETH ERIKSSON AND CLAES JOHNSON
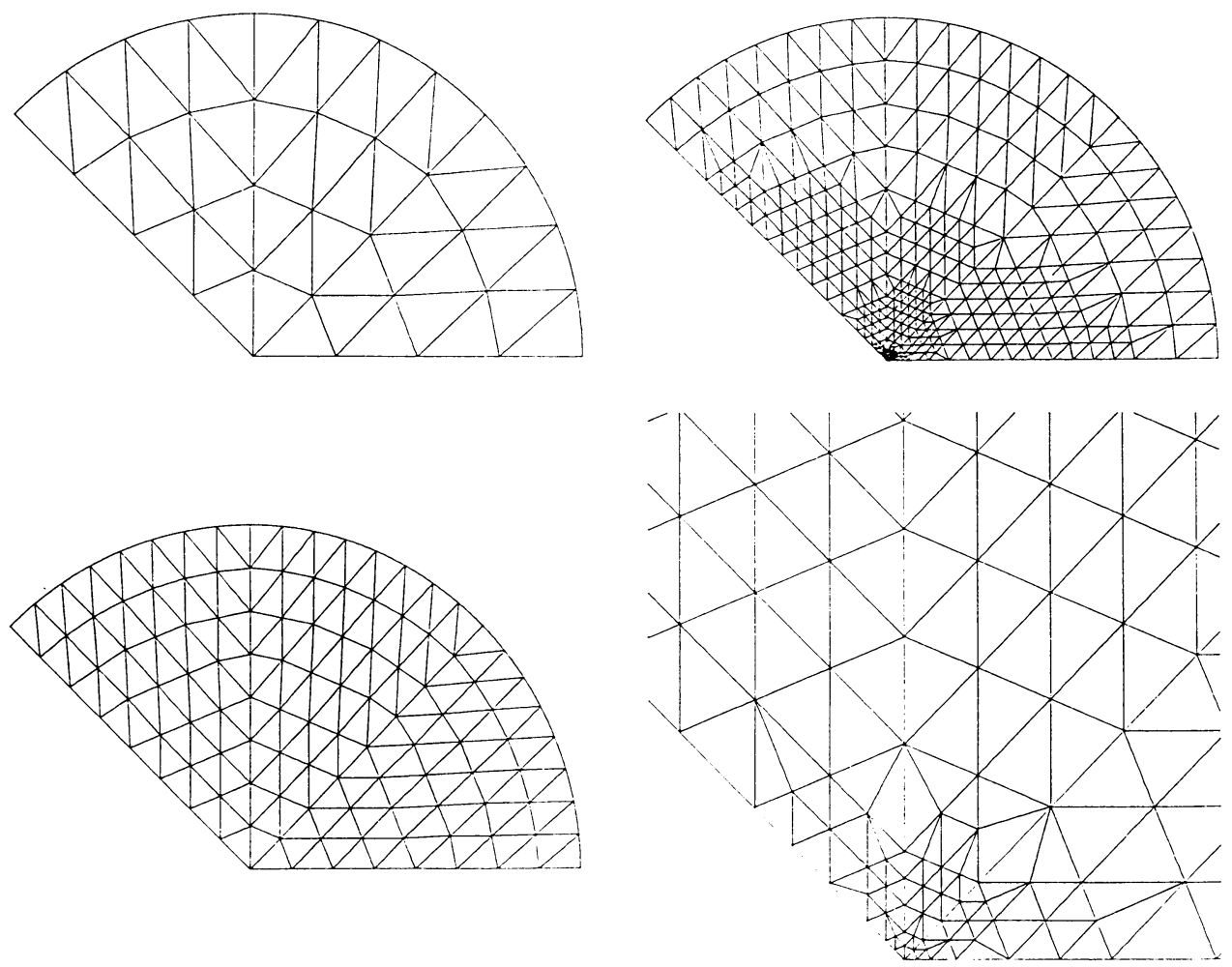

FIGURE 4.3

Magnification 10 times
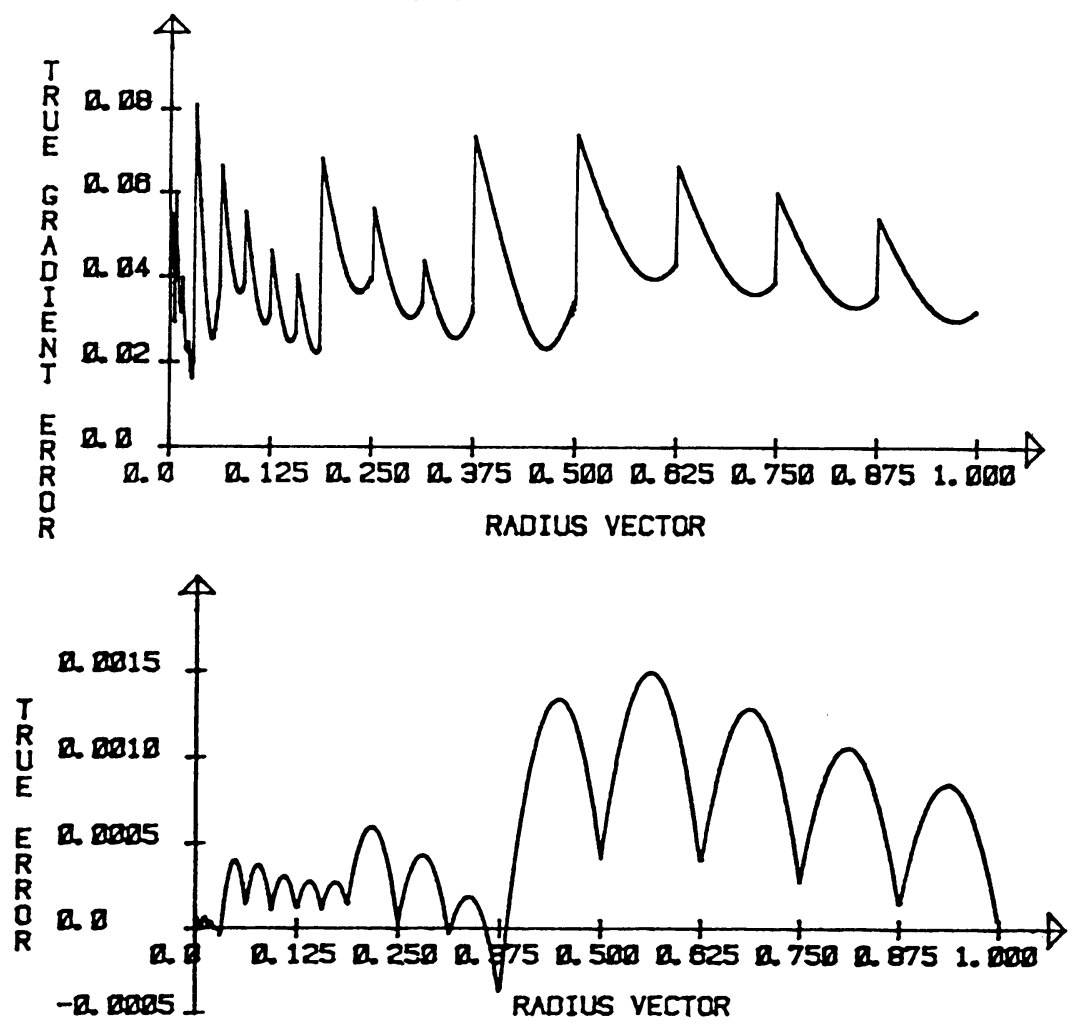

FIGURE 4.4 

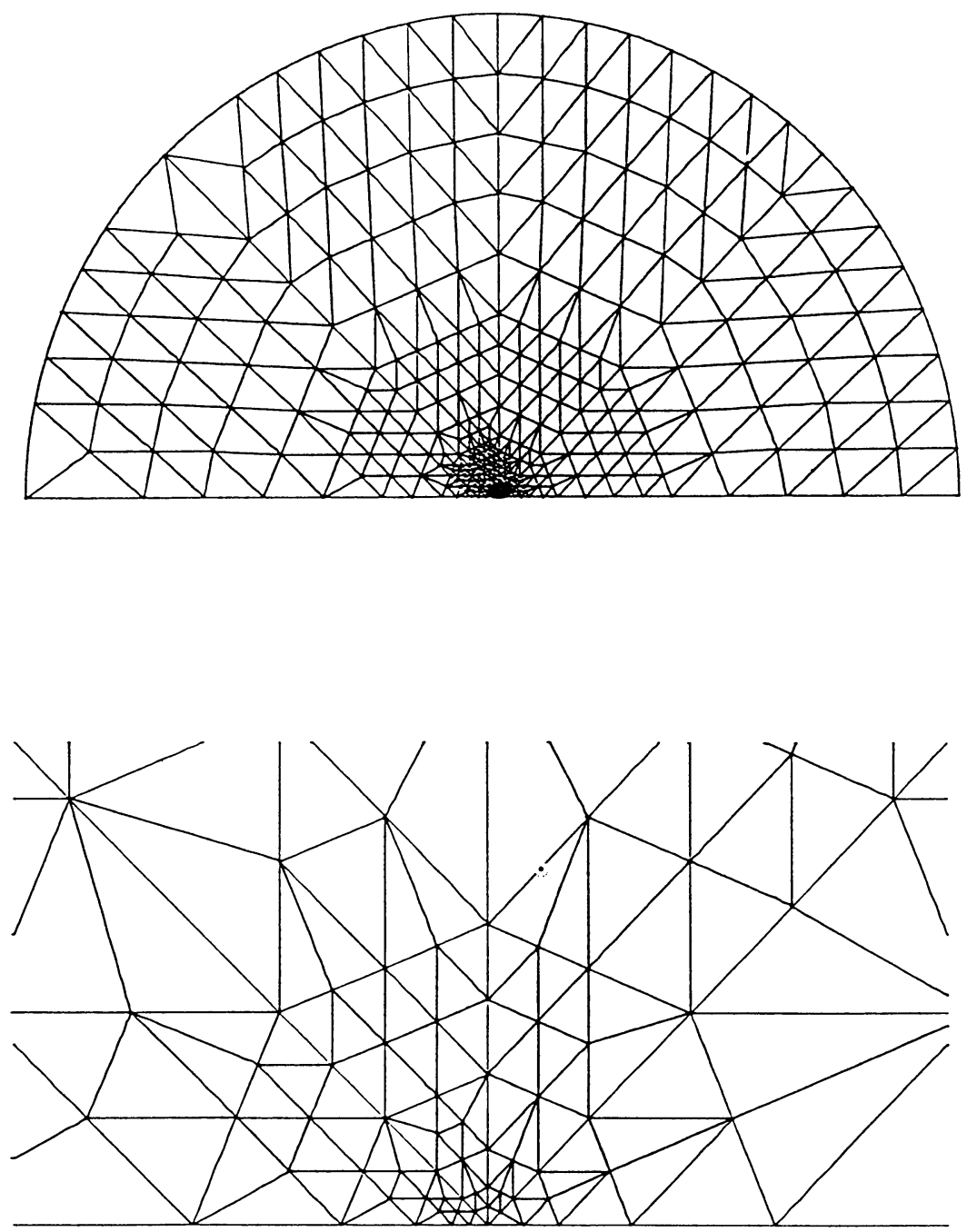

FIGURE 4.5

Magnification 50 times 

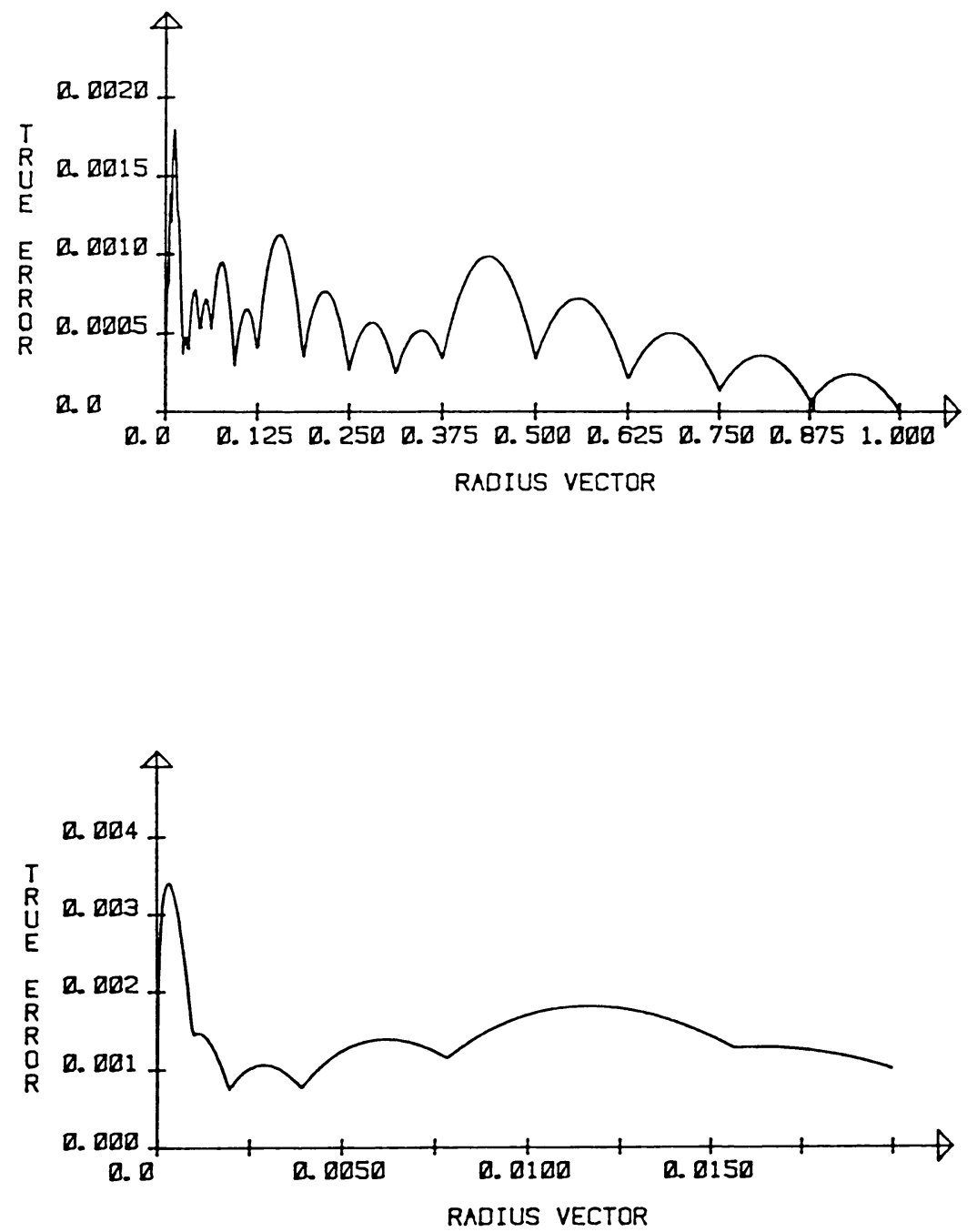

FIGURE 4.6 

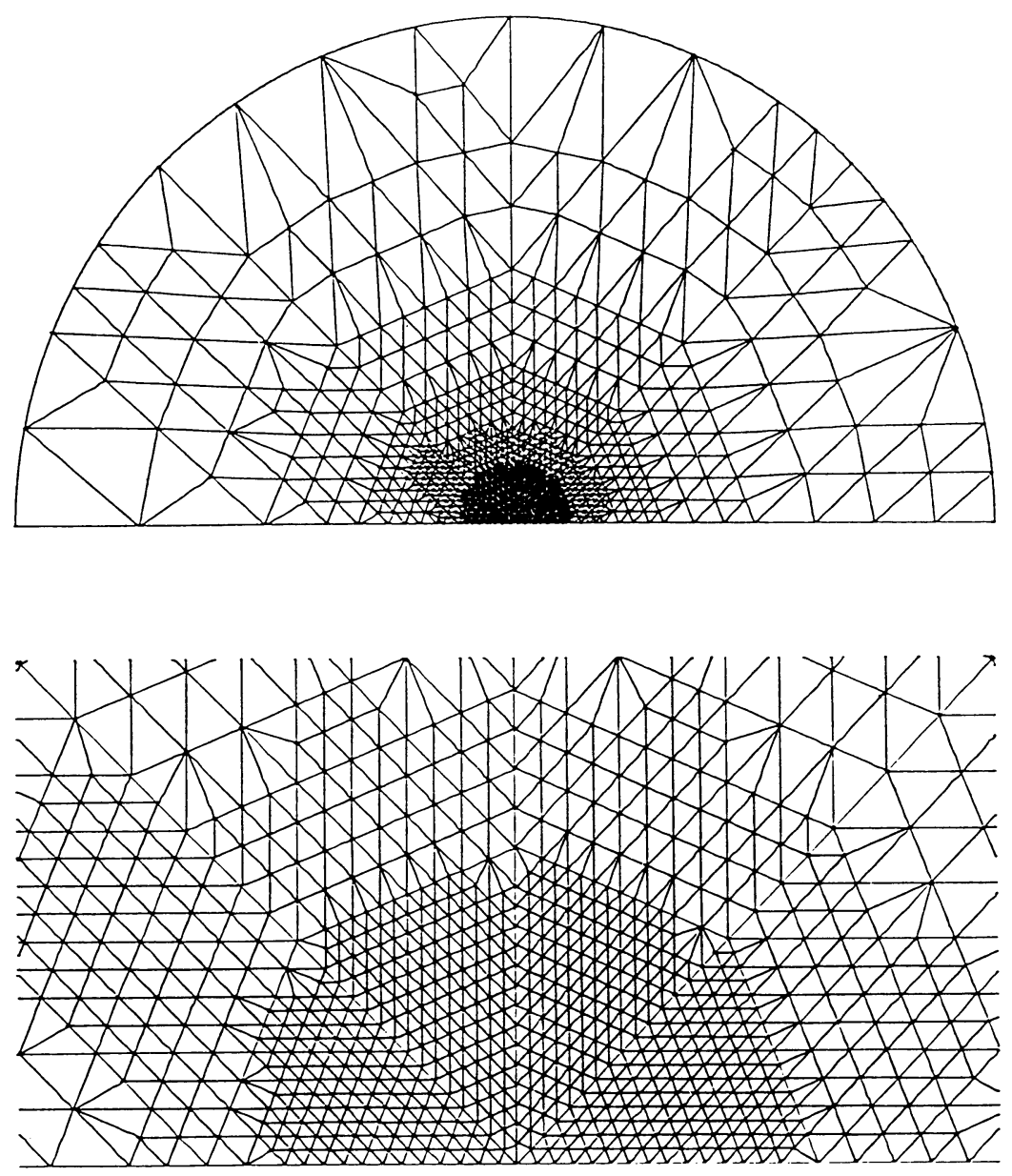

FIGURE 4.7

Magnification 5 times

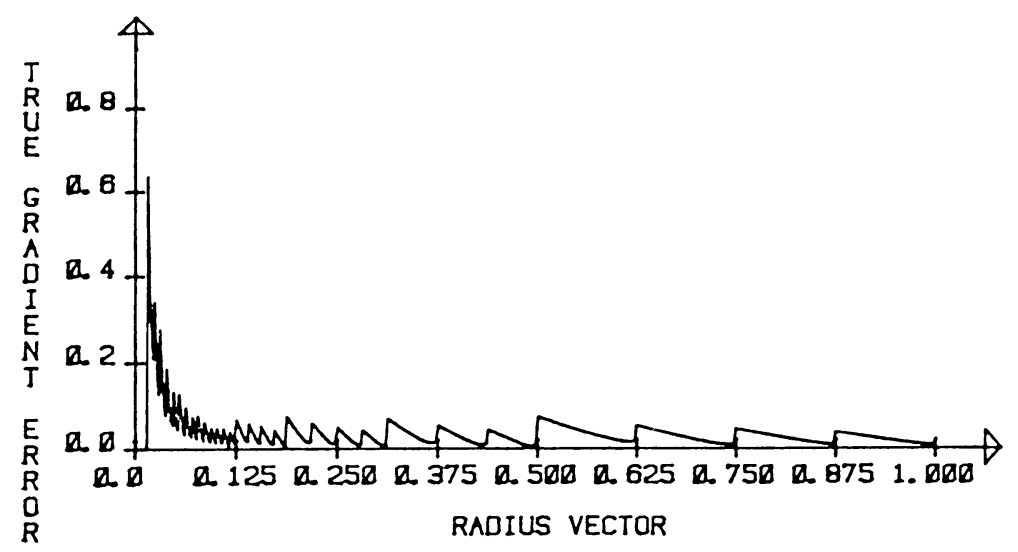

FIGURE 4.8 
Let us now give the results obtained by applying our algorithm to this problem with control of $\|e\|_{\infty, \Omega}$, that is, we simply replace $h_{K}$ and $h_{\tilde{K}}$ in (4.1) and (4.2) by $h_{K}^{2}$ and $h_{\tilde{K}}^{2}$. In Figure 4.5 we give the final mesh, with a zoom at the origin, obtained starting with an initial mesh similar to the initial mesh in Figure 4.3 and using the tolerance $\delta=0.01$. We also give in Figure 4.6 the actual distribution of $|e(x)|$ along the same radius as above, again with a zoom. We observe that $|e(x)|$ is roughly constant in $|x|$ and thus the algorithm succeeds in finding a well-equilibrated mesh.

A theoretical analysis and justification of the algorithm in the present case, which is not covered by this note, since $\beta<1$, is given in [5].

Example 4.3. We also tested the algorithm with control of $|\nabla e(x)|$ for problem (4.3), that is, with control according to (4.1) and (4.2). As already remarked, $|\nabla e(x)| \rightarrow \infty$ as $|x| \rightarrow \infty$ on any mesh, but we now ask how well the algorithm manages to control $|\nabla e(x)|$ for $|x|>0$. In Figure 4.7 we give the final mesh, together with a zoom, obtained by taking $\delta=0.1$. The actual distribution of $|\nabla e(x)|$ is given in Figure 4.8, where we see that in fact $|\nabla e(x)|$ is very well controlled up to the very last elements close to the origin.

Department of Mathematics

Chalmers University of Technology

41296 Göteborg, Sweden

1. F. Angrand, V. Billey, J. Periaux, C. Pouletty \& J. P. Rosenblum, 2-D and 3-D Euler Computations Around Lifting Bodies on Self Adapted Finite Element Meshes, Sixth International Symposium on Finite Element Methods in Flow Problems, Antibes, 1986.

2. I. BABUSKKA, "Feedback, adaptivity, and a posteriori estimates in finite elements: Aims, theory, and experience, Accuracy Estimates and Adaptive Refinements in Finite Element Computations (I. Babuska, O. C. Zienkiewicz et al., eds.), Wiley, New York, 1986.

3. I. BABUSKa \& A. Miller, A Posteriori Error Estimates and Adaptive Techniques for the Finite Element Method, Technical Note BN-968, Univ. of Maryland, 1981.

4. I. BABUŠKA \& A. K. NOOR, Quality Assessment and Control of Finite Element Solutions, Technical Note BN-1049, Univ. of Maryland, 1986.

5. R. E. BANK, PLTMG Users' Guide, June, 1981 version, Technical Report, Department of Mathematics, University of California at San Diego, La Jolla.

6. L. DEMkowics, P. Devloo \& J. T. ODEN, "On an $h$-type mesh refinement strategy based on minimization of interpolation errors," Comput. Methods Appl. Mech. Engrg., v. 53, 1985, pp. 67-89.

7. A. R. DIAZ, N. KIKUCHI \& J. E. TAYLOR, "A method of grid optimization for finite element methods," Comput. Methods Appl. Mech. Engrg., v. 41, 1983, pp. 29-45.

8. K. ERIKSSON, "A maximum norm error estimate for the finite element method for linear elliptic problems under weak mesh regularity assumptions." (To appear).

9. K. ERIKsSON, Adaptive Finite Element Methods Based on Optimal Error Estimates for Linear Elliptic Problems, Tech. Rep. 1987-02, Math. Dept., Chalmers Univ. of Technology, Göteborg, to appear in Math. Comp.

10. K. ERIKSSON, C. Johnson \& J. Lennblad, Optimal Error Estimates and Adaptive Time and Space Step Control for Linear Parabolic Problems, Preprint no. 1986-06, Dept. of Math., Chalmers Univ. of Technology, Göteborg, submitted to SIAM J. Numer. Anal.

11. K. ERIKSSON \& C. JOHNSON, "Error estimates and automatic time step control for nonlinear parabolic problems, I," SIAM J. Numer. Anal., v. 24, 1987, pp. 12-23.

12. K. ERIKSSON \& C. JOHNSON, An Adaptive Finite Element Method for Linear Advection Problems, Tech. Rep., Math. Dept., Chalmers Univ. of Technology, Göteborg. (To appear).

13. R. E. EWING, "Adaptive mesh refinements in large scale fluid flow simulations," Accuracy Estimates and Adaptive Refinements in Finite Element Computations (I. Babuska, O. C. Zienkiewicz et al., eds.), Wiley, New York, 1986. 
14. C. Johnson, Error Estimates and Automatic Time Step Control for Numerical Methods for Stiff Ordinary Differential Equations, Preprint no. 1984-27, Dept. of Math., Chalmers University of Technology, Göteborg; revised version to appear in SIAM J. Numer. Anal.

15. C. Johnson, Y.-Y. NiE \& V. ThomÉE, An A Posteriori Error Estimate and Automatic Time Step Control for a Backward Euler Discretization of a Parabolic Problem, preprint no. 1985-23, Dept. of Math., Chalmers University of Technology, Göteborg, submitted to SIAM J. Numer. Anal.

16. R. LOHNER, K. MORGAN \& O. C. ZIENKIEWICZ, "An adaptive finite element procedure for compressible high speed flows," Comput. Methods Appl. Mech. Engrg., v. 51, 1985, pp. 441-465.

17. R. RANNACHER \& R. SCOTT, "Some optimal error estimates for piecewise linear finite element approximations," Math. Comp., v. 38, 1982, pp. 437-445.

18. A. H. SChatz \& L. B. Wahlbin, "Maximum norm estimates in the finite element method on plane polynomial domains. Part 1," Math. Comp., v. 32, 1978, pp. 73-109.

19. A. H. SChatz \& L. B. Wahlbin, "Maximum norm estimates in the finite element method on plane polygonal domains. Part 2, refinements," Math. Comp., v. 33, 1979, pp. 465-492. 